$\Rightarrow$ GENETICS

\section{CNVs in CAKUT}

Congenital anomalies of the kidney and urinary tract (CAKUT) comprise a broad spectrum of renal malformations. The underlying genetics is complex: a number of monogenic causes exist but can have pleiotropic manifestations. A substantial number of patients also carry copy number variants (CNVs), many of which are associated with genomic disorders (GDs). A new, large-scale study of CNVs has now helped researchers to define distinct genetic landscapes of CAKUT phenotypes.

To assess the contribution of CNVs to CAKUT phenotypes, Simone Sanna-Cherchi and colleagues genotyped rare, large CNVs in 2,824 patients with CAKUT and 21,498 controls. "In addition to demonstrating a high burden of rare CNVs in CAKUT we also defined unique features for CAKUT subcategories," says Sanna-Cherchi. Patients with kidney anomalies had the largest burden of rare CNVs, which were mostly deletions and encompassed known GDs; patients with vesicoureteral reflux had a high burden of CNVs, which were mostly duplications, but a lower burden of GDs; and patients with obstructive uropathy had an intermediate burden of GDs and rare CNVs. "These data indicate that, from a CNV standpoint, different CAKUT categories are genetically distinct," says Miguel Verbitsky, the first author on the paper. "Furthermore, we identified $45 \mathrm{GDs}$ at 37 independent loci, supporting high genetic heterogeneity for CAKUT; however, six of these loci accounted for about $65 \%$ of cases with a GD, thus identifying major regions that are likely to encompass critical regulators of kidney and urinary tract development." Finally, using a mouse allelic series the researchers identified TBX6 as a major driver of CAKUT phenotypes in patients with $16 \mathrm{p} 11.2$ microdeletion syndrome - one of the most common genetic causes of autism.

Sanna-Cherchi explains that the identification of variants with incomplete penetrance and variable expressivity of phenotypes provides a framework for studying these fundamental aspects of human genetics. "By understanding these complex aspects we can understand the mechanisms by which certain individuals can compensate and tolerate mutations, whereas others progress to end-stage renal disease, and envision the development of novel tools to mitigate the effect of human mutations," he says.

Susan J. Allison

ORIGINAL ARTICLE Verbitsky, M. et al. The copy number variation landscape of congenital anomalies of the kidney and urinary tract. Nat. Genet. 51, 117-127 (2018)

\title{
GENETICS
}

\section{The genetic architecture of blood pressure}

A multi-omics study by Adriana Hung, Todd Edwards and colleagues provides new insight into the genetic basis of blood pressure (BP) traits. "This work was motivated by important advances in the field that have enabled genetic investigations to be more comprehensive, including the availability of large cohorts and tissuespecific reference panels of gene expression," explains Hung.

First, the researchers performed a genome-wide association study (GWAS) of data from the Million Veteran Program (MVP) and the United Kingdom Biobank with independent replication in other large cohorts. These analyses, which included data from $>750,000$ individuals, identified 208 novel common single-nucleotide polymorphisms (SNPs) and 53 novel rare variants that were associated with BP traits.

To evaluate the relationship between genetically predicted gene expression (GPGE) levels and BP traits, the researchers conducted a transcriptome-wide association analysis using the S-PrediXcan method. They identified $>4,000$ BP associations with predicted expression of 840 genes in
45 tissues. A phenome-wide association study of BP genetic risk scores in the MVP cohort showed that the effects of common SNPs on $\mathrm{BP}$ were consistent between ethnic groups.

The researchers also analysed the GPGE data to identify gene-drug relationships. "We found 41 potential novel gene targets for BP treatment and 289 gene targets for existing drugs that could potentially be repurposed as antihypertensives, including several drugs that target kidney BP genes," says Edwards.

In addition, they used single-cell sequencing of mouse kidney cells to investigate the expression of homologues of genes that were identified as significant in the S-PrediXcan analysis of kidney tissue. "We observed evidence that tubules are the key structures in the kidney that mediate genetic influences on BP," comments Edwards.

The researchers are now conducting a much larger trans-ethnic GWAS of BP traits that includes additional data from East Asian and Eastern European populations.

Ellen F. Carney

ORIGINAL ARTICLE Giri, A. et al. Trans-ethnic association study of blood pressure determinants in over 750,000 individuals. Nat. Genet. 51, 51-62 (2019)

\section{DEVELOPMENT}

\section{Some nephron progenitors fail to commit}

Nephron progenitor cells (NPCs) are thought to undergo a linear progression from an uncommitted to a fully committed state as they move from the top of the cap mesenchyme to the junction between the tip and stalk of the ureteric epithelium where nephron formation occurs. Now, Alexander Combes, Melissa Little, Kynan Lawlor and colleagues report that not all NPCs that express commitment genes immediately form nephrons.

"We previously showed that the cap mesenchyme behaves like a randomly moving swarm," says Combes. "Our current study attempts to understand how NPCs can exist as a randomly moving population but simultaneously undergo commitment to form nephrons at defined sites."

As Wnt4 is one of the earliest known markers of progenitor commitment, the researchers used Wnt4 lineage tracing, live microscopy and single-cell RNA sequencing to investigate NPC differentiation in mice. They report that most NPCs that express Wnt4 commit to nephron formation but a small proportion "escape" commitment and migrate back into the uncommitted population, where they are able to return to a progenitor-like state.

These findings were consistent with a computer simulation in which commitment occurred in response to a spatially restricted induction signal in a motile progenitor population. "Cells that reached a certain threshold of induction signal committed, but those that moved away before this threshold was reached could return to the uncommitted population," explains Lawlor.

"Having a stochastic element to commitment may be an efficient and simple way to maintain a progenitor population that is constantly moving but simultaneously giving rise to committed cells at a precise location," suggests Combes. "This mechanism may be important to maintain the reciprocal signalling between the nephron progenitors and underlying tip that drives kidney growth through branching morphogenesis."

Ellen F. Carney

ORIGINAL ARTICLE Lawlor, K. T. et al. Nephron progenitor commitment is a stochastic process influenced by cell migration. eLife 8, e41156 (2019) 